\title{
Social-aware Event Handling within the FallRisk Project
}

\author{
Femke De Backere'; Jan Van den Bergh'²; Sven Coppers²; Shirley Elprama33; Jelle Nelis'; \\ Stijn Verstichel'; An Jacobs ${ }^{3}$; Karin Coninx ${ }^{2}$; Femke Ongenae ${ }^{1}$; Filip De Turck ${ }^{1}$ \\ 'Information Technology Department (INTEC), Ghent University - iMinds, Ghent, Belgium; \\ 2Expertise center for Digital Media (EDM), Hasselt University - iMinds, Diepenbeek, Belgium; \\ ${ }^{3}$ Studies on Media, Information and Technology (SMIT) - Vrije Universiteit Brussel - iMinds, Brussels, Belgium
}

Keywords

Decision making, computer-assisted, decision support systems, information systems, usercomputer interface

\section{Summary}

Objectives: With the uprise of the Internet of Things, wearables and smartphones are moving to the foreground. Ambient Assisted Living solutions are, for example, created to facilitate ageing in place. One example of such systems are fall detection systems. Currently, there exists a wide variety of fall detection systems using different methodologies and technologies. However, these systems often

Correspondence to:

Femke De Backere

Department of Information Technology

Internet Based Communication Networks

and Services (IBCN)

Ghent University - iMinds

Technologiepark 15

B-9052 Gent

Belgium

E-mail: femke.debackere@intec.UGent.be do not take into account the fall handling process, which starts after a fall is identified or this process only consists of sending a notification. The FallRisk system delivers an accurate analysis of incidents occurring in the home of the older adults using several sensors and smart devices. Moreover, the input from these devices can be used to create a socialaware event handling process, which leads to assisting the older adult as soon as possible and in the best possible way.

Methods: The FallRisk system consists of several components, located in different places. When an incident is identified by the FallRisk system, the event handling process will be fol-

Methods Inf Med 2017; 56: 63-73 https://doi.org/10.3414/ME15-02-0010 received: November 1, 2015 accepted in revised form: November 9, 2016 epub ahead of print: December 6, 2016

Funding

The iMinds FallRisk project is cofunded by iMinds (Interdisciplinary Institute for Technology), a research institute founded by the Flemish Government. lowed to assess the fall incident and select the most appropriate caregiver, based on the input of the smartphones of the caregivers. In this process, availability and location are automatically taken into account.

Results: The event handling process was evaluated during a decision tree workshop to verify if the current day practices reflect the requirements of all the stakeholders. Other knowledge, which is uncovered during this workshop can be taken into account to further improve the process.

Conclusions: The FallRisk offers a way to detect fall incidents in an accurate way and uses context information to assign the incident to the most appropriate caregiver. This way, the consequences of the fall are minimized and help is at location as fast as possible. It could be concluded that the current guidelines on fall handling reflect the needs of the stakeholders. However, current technology evolutions, such as the uptake of wearables and smartphones, enables the improvement of these guidelines, such as the automatic ordering of the caregivers based on their location and availability.

\section{Introduction}

As the life expectancy in Europe has steadily been risen in the past decades [1], the older adults population has grown to new heights [2]. In 2014 the total population of the EU-28 was estimated at 508,6 million, with a share of $18.5 \%$ of people aged 65 or over [3]. Eurostat uses an indicator Healthy Life Years at birth, which measures the number of years that a person is expected to live in healthy conditions [4]. In 2013, this number was estimated at 61.4 years for men and 61.5 years for women.
When this is compared to the life expectancy at birth, this represents respectively $79 \%$ and $74 \%$ of the life expectancy at birth. This indicates that older adults live in some type of disability for some years at the end of their lives.

Studies have indicated that about $90 \%$ of persons aged 55 and older would prefer aging in place for as long as possible [5], mostly because being independent is very important to them. 'Aging in place' [6] can be defined as remaining living in the community, with some level of independence, rather than in residential care. However, to make this possible, these older adults are dependent on a wide range of caregivers, ranging from informal caregivers, such as their relatives, to formal caregivers, such as (home care) nurses, general practitioners and other healthcare professionals [7]. Moreover, to facilitate care at home for these caregivers, but also for the older adults, there is a need for assistive technology. Assistive technology has proven to ease the burden especially on informal caregivers [8].

One of the major causes of mortality and morbidity in older adults are fall inci- 
dents [9]. These incidents may lead to the inability to stay in the community and being admitted in a nursing home. Falling also leads to a decline in the ability to perform Activities of Daily Living (ADL), requiring more care if they are able to remain at home. Consequently, fall incidents may increase the burden on the (in)formal caregivers. Studies reveal that older adults experience assistive technology with respect to fall incidents positive, as it can promote healthy active aging and more important independence and control. However, these technologies should be kept as simple, effective and tailored to the individual needs [10].

Nowadays, older adults, aging in place, are often equipped with a Personal Emergency Response System (PERS). A PERS often consists of a button that can be pressed and then will connect to an emergency call center. At this center, a desktop operator is able to talk to the older adult and assess whether assistance should be sent [11]. However, problems arise with such systems. First, the older adults are required to wear the PERS at all occasions in the home, (ii) the older adult might be reluctant to press the button when something happens as they do not want to burden caregivers, (iii) the systems is often triggered by accident or triggered when no incident occurred and (iv) there is no assistance available when the older adult moves out of the house.

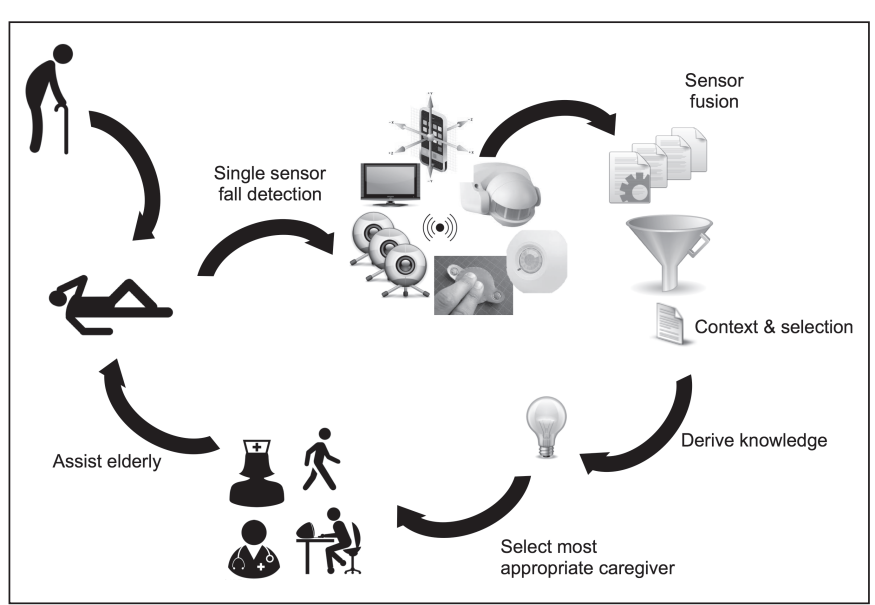

Figure 1 Objectives of the FallRisk system. When a fall incident occurs, the fall is picked up by several single sensor systems. Sensor fusion will combine the single sensor inputs and will filter false positives and false negatives. By adding context to the fall incident a better understanding can be given to the data. The derived knowledge is used to trigger an alarm and the most appropriate caregiver is selected to assist the older adult.
When the desktop operator receives an emergency call, a caregiver is notified, if necessary. The selection of the caregiver is made based on a fixed list, which is composed when the PERS is taken in use. This fixed list leads to a number of disadvantages, such as the unavailability of the caregiver due to work or vacation or the distance the caregiver is removed from the emergency scene.

With the uprise of the Internet of Things and pervasive healthcare, wearables and smartphones, these personal systems could be improved $[12,13]$. Nevertheless, issues surface when implementing such systems [14, 15], such as the quality of the sensors, the trustworthiness and the power consumption. To tackle these issues, the FallRisk project ${ }^{*}$ focusses on using different fall detection systems and combining the results of the single sensors to come to more accurate decisions. To provide a more flexible solution for notifying the most suitable caregiver, algorithms are developed to take into account availability and distance from the caregiver to the older adult. To facilitate communication between all parties, namely the older adult, the caregivers and the desktop operator, userfriendly smartphone and tablet applications are developed. As literature indicates

http://www.iminds.be/en/projects/2014/03/19/ fallrisk that ambient assisted living (AAL) solutions should be based on the requirements and needs of the involved stakeholders [16-18], this FallRisk system and the accompanying algorithms were designed while incorporating the feedback of these stakeholders.

Literature indicates that a lot of attention already has been given to the detection of falls using single sensor systems. However, the next steps after the fall detection, namely notifying caregivers and the assignment to offer assistance as soon as possible are often not researched. In this research, focus is given to how a fall event should be handled in the best manner using, smartphone and tablet application and taking context into account.

The remainder of this paper is structured as follows. Section 2 details the objectives of the FallRisk system. To make the original contribution of this research clearer, Section 3 gives an overview of the current state of the art, whereas Section 4 deals with the methodological approach. Section 5 details the evaluation and results of the event handling process. Finally, the results are discussed in Section 6 and the conclusions are highlighted in Section 7.

\section{Objectives}

The aim of this research is to design a social-aware event handling system, with special focus on handling fall events. This system enables the following features:

- Collection of information from single sensor fall detection systems and fuse these pieces of sensor information to create accurate knowledge on the fall incident.

- When the system concludes that a fall occurred, the system decides upon which caregiver is the most appropriate person to help the older adult. This decision is based on context information gathered by the apps used by the older adult and caregivers.

- Assist the caregiver, desktop operator and older adult during the process using smartphone and tablet applications.

The features of the systems are visualized in Figure 1 . The original research con- 
tribution of the paper is the design of a system where fall incidents are handled in a social-aware way, taken into account the above-mentioned features. Within this paper, focus is especially given to the event handling process after a fall incident trigger is received. This way, help will be given to the older adult as fast as possible, which will minimize the effects of the fall. The design of the process is outlined in this paper together with the evaluation of the algorithm.

\section{Related Work}

The design and development of fall detection systems have seen an increase the past years, as is indicated by the multitude of literature reviews and systematic studies. On the one hand, these reviews focus on fall detection systems as such and the different technologies and methodologies used to realize trustworthy, reliable and accurate fall detection [19-21]. On the other hand, attention is given to how older adults perceive and use these systems [22, 23]. However, little attention is given to what happens after a fall is detected and what is the most efficient way to handle a fall.

Not only the accurate detection of a fall is important when older adults are living independently at home, also minimalizing the time, needed to intervene and assist, is of the utmost importance. Studies have indicated that older adults lying too long on the floor, e.g., after a fall, may experience fear of falling, muscle damage, dehydration and even death in the long end [24,25]. Moreover, a fall incident can lead to the inability to keep living independently at home and being admitted to an institutional care facility [26].

To the best of our knowledge, fall detection systems currently only notify one or multiple (in)formal caregivers or a call center or at least literature does not further describe the handling of a fall in detail. Most of the times, this notification is sent as a text message, mail or smartphone notification, indicating that something happened in the home of the older adult [27-30]. Using a text message or mail as a means of notification, however, makes it difficult to detect whether the receiver of the message will act upon this message. Most of the times, these smartphone-based systems have no integration with existing PERS systems and caregivers, mentioned within the system, are notified all at once. This increases the burden on the informal caregivers. In the system, proposed by Terroso et al., the GPS position of the fall is added to the text message [31].

Based on our research of the current state of the art, we can conclude that little attention has been given to how a fall can be handled in such a way that the older adult is offered assistance as fast as possible and in the most appropriate manner. The remainder of this paper will therefore first give a brief overview of the components within the FallRisk system and most importantly detail the fall handling process when the system has detected a fall.

\section{Methods}

The FallRisk system facilitates sensor fusion and the social-aware acting upon the decisions made by the reasoning mechanisms. Section 4.1 details the general concept of the system, whereas Section 4.2 specifies a scenario, to make clear how the system works from an end user's perspective. Section 4.3 focuses on the specific components of the FallRisk system and their interactions. Finally, Section 4.4 highlights the event handling process used within the FallRisk system and the differently designed apps for the older adult, the caregivers and the call center, which are used on the one hand as a communication means and on the other hand for gathering the necessary data.

\subsection{General Concept}

The general concept of the FallRisk system is illustrated in - Figure 2. As can be seen in this figure, data from two specific types of end users are taken into account, namely, data from the older adult and data from the caregivers. The data from the older adult can be split into two different types. First, there is the data from the sensors, which are used within the home setting. Second, context information of the older adult is made available, for example, the presence of carpet in the home or previous fall incidents of the older adult. The data from the caregivers mostly consists of data concerning their availability and location, as this information will be taken into account when something happens with the older adult. All this data is collected into the cloud, where the data can be combined and sensor information, from a certain time span, can be used to detect falls. When the FallRisk system actually detects a

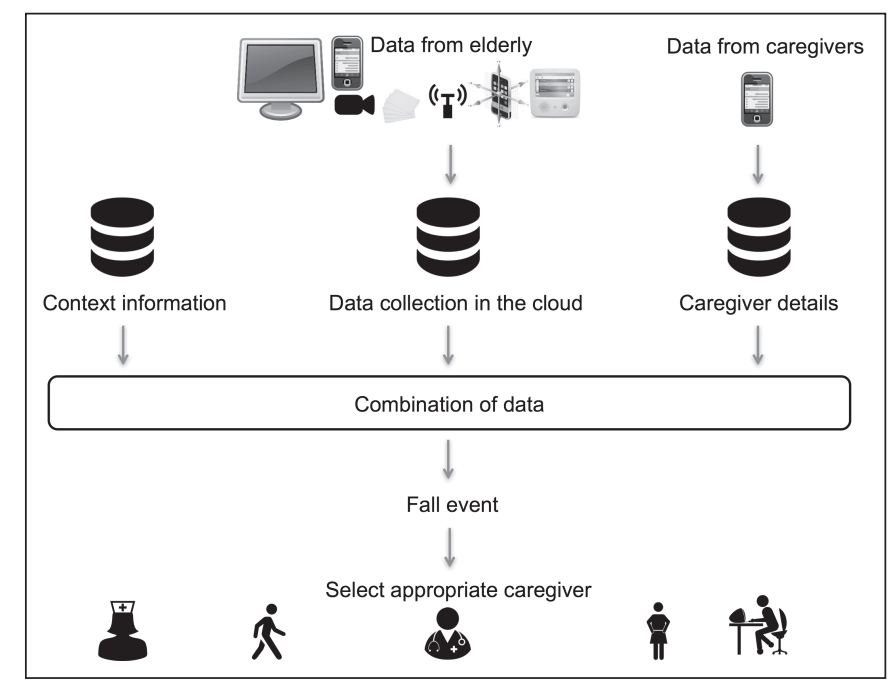

Figure 2 The general concept of the FallRisk system. Data from the older adult, such as sensor data, localization and context information is gathered in the cloud, together with data from the caregiver, both static and dynamic. All this data is combined in the cloud and if a fall event is triggered, the most appropriate caregiver can be selected. 
fall, the most appropriate caregiver can be selected based on the location and availability data from the (in)formal caregivers.

\subsection{Scenario: A Day with Falling}

The previous section discussed the general concept of the social-aware event handling system, in this section a scenario example is given to detail how the system works from an end user's perspective.

Mary is 82 years old, living alone. When her husband died a few years ago, her family decided to install the FallRisk system to enable her to stay at home. Mary's home was installed with a number of fall detection systems and other sensors, namely a camera system, Passive InfraRed sensors (PIRs), pressure sensors in the couch and bed, door/window contacts on every door and window and shout detection. Mary also has a smart TV, which is coupled to the FallRisk system, as well as a terminal which is used as the interface with her PERS. Mary now also has a new smartphone. This smartphone is equipped with an app using the accelerometer to detect falls.

1. One day, Mary is in the living room, but standing up too quickly to go to the kitchen, she loses her balance and falls on the floor. She cannot get up and starts shouting in panic.

2. The sensors, camera and smartphone identify a possible fall and also Mary's shouting is detected. The FallRisk system handles the information and concludes that a fall has occurred.

3. Mary can view on the screen within her house, namely the smartphone, TV and terminal, that the system has detected a fall. The system asks her whether something happened. As Mary is not answering this questions within 10 seconds, the desktop operator Alice in the call center is notified.

4. Alice takes a look at the data and information on the screen. The FallRisk system will automatically initiate a call between Alice and Mary. Alice fills in the checklist to assess the fall incident and asks Mary if she needs assistance, which is the case.

5. This means that Alice has to contact the caregivers of Mary. She checks the availability of Mary's caregivers and based on the information gathered from the smartphone, she can see that Ann is the most suited caregiver to assist Mary at the moment. Ann also has key access to the home.

6. Alice contacts the informal caregiver Ann if she can help Mary. Ann receives

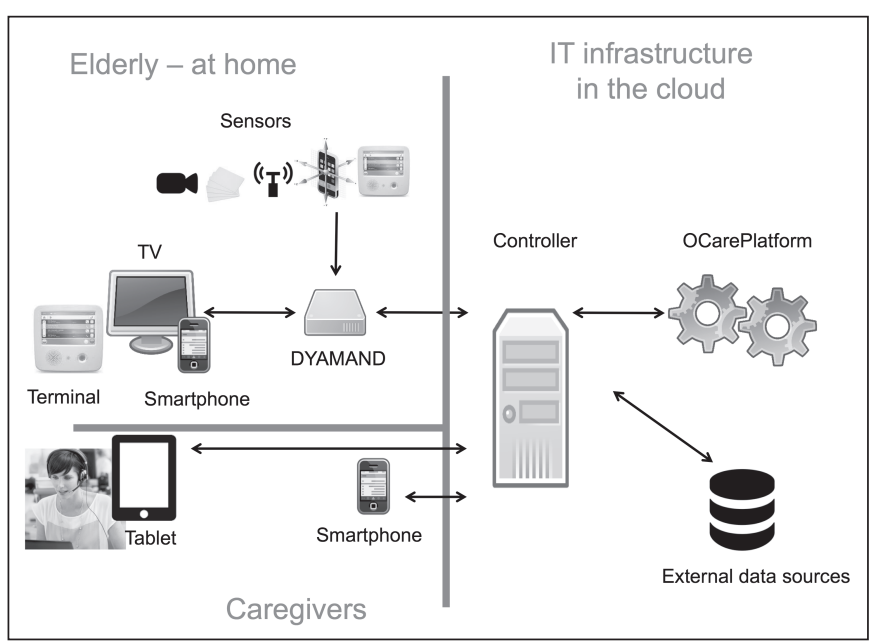

Figure 3 The FallRisk components and their interactions. The sensors in the house of the older adult, together with the smart devices, such as the terminal, smart TV and smartphone, will send their information to a gateway, which is running the DYAMAND framework. DYAMAND will format the data and send it through to the cloud, where the Controller is responsible for sending the data to the OCarePlatform. The OCarePlatform will use reasoning techniques to derive knowledge and will send notifications to the Controller. The Controller will then decide what to do with these notifications. If communication has to be set up between the mobile applications, the Controller will notify the correct device. a message on the smartphone and indicates that she can be of assistance.

7. During this process, Mary is kept up to date on the progress by her smartphone, the TV and the terminal. When Ann accepts the task to help Mary. Mary is notified using these smart devices in her home that Ann will arrive within 15 minutes.

8. Once arrived at Mary's place, Ann helps Mary up, but Mary does not feel that well and Ann helps her in the armchair to take a rest. Ann registers herself on the terminal, to notify the desktop operator that she has arrived.

9. Afterward, there is a follow-up protocol to see if Ann was able to help Mary.

\subsection{FallRisk Components and Interactions}

To realize the FallRisk system, the system was split up into different components, each with their own responsibilities and functionality. The high-level overview of the components' break-down is visualized in $>$ Figure 3 . The following subsections will detail each component.

\subsubsection{Devices and Sensors within the Home Setting of the Older Adults}

The home of the older adult is equipped with a considerable amount of different types of sensors. This set-up can be specified to the specific needs of the older adult or the lay-out of the house. There is no required set of sensors that should be deployed. Other systems that can be coupled to the system are (i) a camera-based fall detection system, (ii) a terminal, with a scream detection system, interfaced to the PERS, which then can be used to connect to the call center and (iii) a smartphone, where internal sensors (e.g. the accelerometer [32]) can also be used for detecting falls.

There are three ways to facilitate communication with the older adult in case something happens: (i) the smartphone, using the elderly app, (ii) a smart TV and (iii) the terminal, this is a device equipped with sensors and a registration system for caregivers. 


\subsubsection{DYAMAND}

These sensor and systems are all connected to a gateway, running DYAMAND [33]. DYAMAND (Dynamic, Adaptive MAnagement of Networks and Devices) is responsible for sending all this data, gathered within the home of the older adult, to the cloud. The DYAMAND framework acts as a middleware layer between connected devices and the cloud, where all data is gathered.

\subsubsection{The Controller}

The Controller functions as the access point to the cloud. The Controller functions as a gateway between DYAMAND and the OCarePlatform. The Controller is also the mediator between the smartphone and tablet applications of the older adult, the caregivers and the call center.

\subsubsection{The OCarePlatform}

The next component in the cloud is the OCarePlatform. The OCarePlatform [34] is a data-driven platform, which receives all the information from the FallRisk system. The OCarePlatform is developed in such a way that intelligent, semantic services can be added in a straightforward manner to solve specific use cases, for example, the detection of fall events. It does not matter if some information, which is sent to the OCarePlatform, is not relevant. By using the Semantic Communication Bus (SCB) [35], the relevant information is sent to the services, which have indicated a specific interest in that type of data.

As often false positives and negative are generated from single sensors or fall detection systems, the OCarePlatform is used to combine all this information and come to 1 conclusion. This is done by combining the information from the different sensors and fall detection systems. Within the OCarePlatform, it is also possible to take external information into account. The knowledge, derived by the system, is sent to the Controller. The Controller will send the information to the correct device, based on the specific type of information.

\subsection{Event Handling Process}

When the OCarePlatform triggers an alarm, the call center app is triggered. This app is key within the event handling process. This process is responsible for deciding how the fall event is handled. In order to do so, the call center app is communicating with two other apps, namely the caregiver app and the elderly app. In the following sections, the call center app of the desktop operator, the caregiver app of the (in)formal caregivers and the elderly app of the older adult are discussed and finally the details of the event handling algorithm are detailed.

\subsubsection{Call Center App}

The PERS, which is currently the most suitable solution for older adults with a fall risk indication, connects to the call center in case the button of the PERS is pressed. It is important that the FallRisk system also initiates the contact between the call center, more specifically the desktop operator and the older adult, because checklists are used to evaluate the severity of the incident. Therefore, the PERS call center can be extended with the call center app.

This app is specifically designed for tablets and is visualized in Figure $4 \mathrm{a}$. The main view of this app consists of three parts. The first part, on the left hand side, gives a general overview of the older adult, e.g., the age of the person, the address, aids used in the home (for example, a stroller), specific context information and important medical information (such as the older adult is a diabetic). The middle part of the application focuses on the questionnaire, which is used during the call. Based on this checklist, the desktop operator is able to assess the fall incident and determine whether an ambulance is be notified. Moreover, sensor information from the home is shown, as this can be used to give further
Figure 4

Call center interface during call (top), main older adult user interface (left under), a preliminary caregiver screen to specify time to arrival (right under). (a) The call center app consists of three parts: (i) older adult information, (ii) information on the incoming call and (iii) the ordered list of caregivers. (b) The elderly app where the older adult can trigger an alarm, call with a caregiver or indicate their mood. (c) An example of the caregiver app. The caregiver can indicate how long it will take to get to the older adult in case of an emergency.

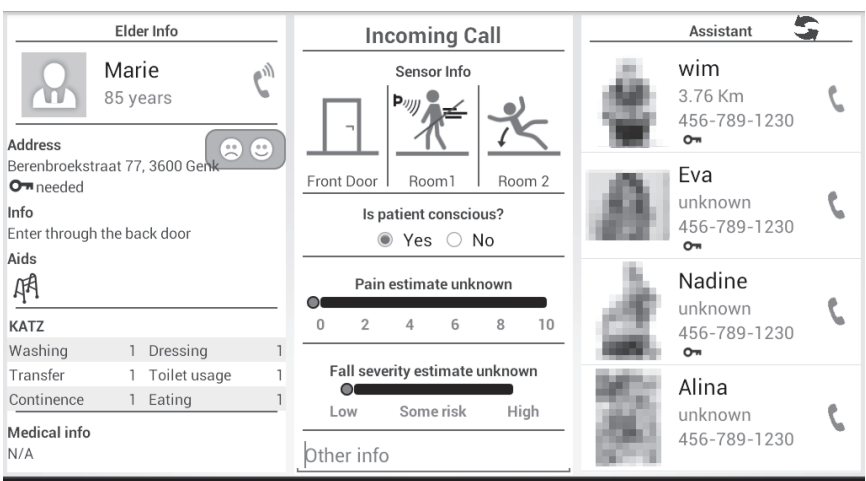

a
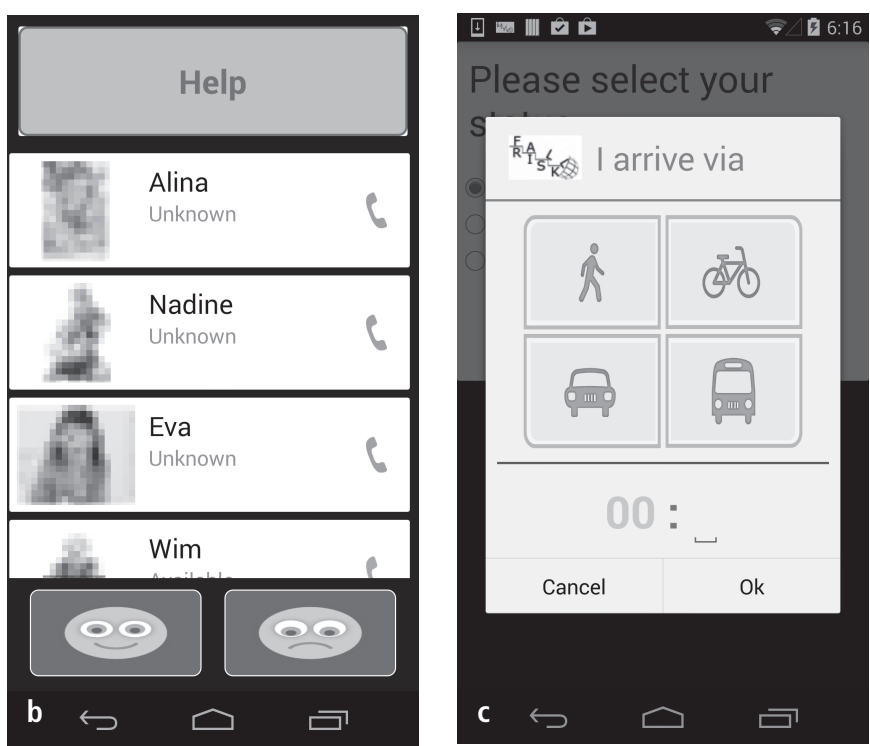
insights into the incident. Finally, the right side of the applications gives an overview of the (in)formal caregivers in the care circle of the older adult. The order of the list is determined based on their location and availability. Extra information about each specific caregiver is shown: name, availability, contact details, location and whether the person has key access to the home of the older adult. The availability is indicated by the telephone icon. The instant messaging protocol of XMPP can be used between the call center app and the other apps to send textual messages.

This call center app has a built-in event handling algorithm, which is used whenever the OCarePlatform triggers a fall within the home of the older adult. This event handling process is further detailed in Section 4.4.4 and uses the data gathered by the caregiver app, discussed in the next section, to determine who to notify.

\subsubsection{Caregiver App}

- Figure $4 \mathrm{c}$ visualizes the caregiver app used by the (in)formal caregivers. These caregivers have the possibility to indicate their availability. This can be specified by being available, unavailable or busy. When the caregiver indicates busy, the call center app will still be able to contact the caregiver, but the older adult cannot contact them. The caregiver is also able to initiate calls and receive calls with the app.

\section{Context-Rule}

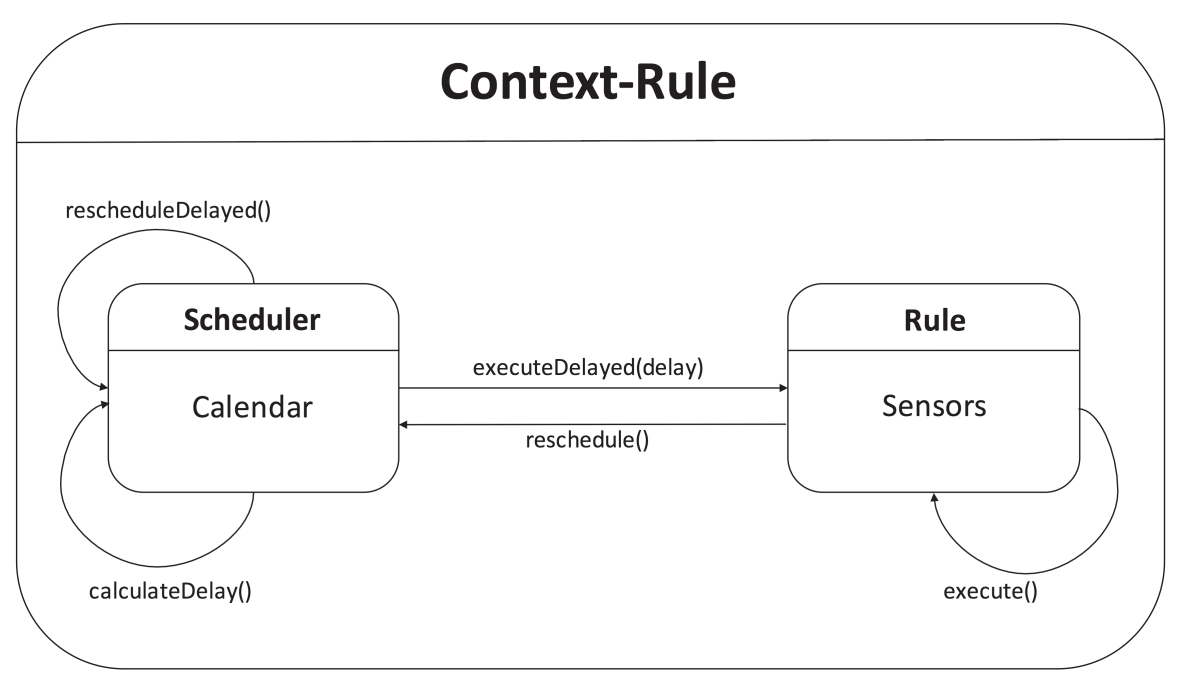

Figure 5 Generic mechanism of context rules used within the smartphone applications of the older adult, the caregiver and the desktop operator.

The location of the caregiver is also automatically detected by the applications and is used when the distance between the older adult and the caregiver needs to be calculated in case of an incident. It is possible to configure whether the presence of a calendar item means being busy ("I am busy but I can go to a fall incident") or unavailable ("I cannot go to a fall incident"). When there is no appointment in the agendas, the caregiver is assumed to be available. Caregivers can also specify their availability manually, with reminders to reset their availability. The integration of reminders is based on testimonials of nurses working at the call center [36].

These calendars are also used by a generic mechanism, as shown in $>$ Figure 5 , to gather context information and notify caregivers (relative location and availability). This mechanism uses a set of schedulers that use calendar information to trigger activation (executeDelayed(delay)) of a context rule, which activates smartphone sensors and triggers the corresponding functionality (execute()). When the rule is executed, the scheduler is requested to plan a new execution of the rule (reschedule()).

For the caregiver app, these rules are used to determine how caregivers are notified in case of an alarm (with sound/vibration/colored notification light/no specific mechanism). The notification preferences may differ based on the availability infor-

Methods Inf Med 1/2017 mation (available, busy, not available); when caregivers are available, they want to be sure not to miss an alarm, which can thus use the default settings of both sound and vibrations. While busy, they may want to be notified in a subtle manner (vibration or even only a colored led during a meeting if the phone is face-up, but with the screen off, on a meeting table). Interviews with informal caregivers [37] indicated that they still want to be informed when a fall incident happens. In this case, a notification can still be sent, but without any additional means to attract attention; i.e. no sound, no vibration.

Context rules are also triggered by external events, such as a detected fall, and trigger specific actions: start measuring location when the caregiver is available or busy and if it was not already turned on and stop after the event if it was off. For the person that agrees to attend to a fall, highaccuracy location tracking is turned on until the moment the fall handling ends. The app does not communicate the absolute location to the call center, but the distance to the older adult as computed through the Google Maps API. Location tracking is also used to verify arrival at the older adult and to notify the caregiver of the need to fill out a small report about the fall when leaving. All three usages of location data are based on problems reported by nurses operating the call center [36]; home address is nowadays frequently not a reliable basis to determine caregiver location, occasional problems were reported by informal caregivers saying they went to an older adult, while in reality, they did not, and closing reports are not always filled out. The corresponding reminder is therefore also a sticky notification; it is only removed once the report is received at the call center.

The caregiver app assists its user to specify the time it takes to get to the older adult if desired; based on the specification of the transportation means, a suggestion is made of how long it takes to reach the older adult. This suggestion can be adapted to take into account issues not related to traffic. The time remaining to arrival is communicated to the older adults. 


\subsubsection{Elderly App}

The elderly application is designed for smartphones and is illustrated in $>$ Figure $4 \mathrm{~b}$. One of the most important features of the app is that calls from the call center are answered automatically, this way the desktop operator can assess the severity of the incident. The app also can be used to request assistance, for example, after a fall. The older adult can indicate their mood on a daily basis. All this information can also be used by the OCarePlatform to take into account.

The app can also be used to initiate calls between the older adult and caregivers in less urgent situations. The older adult will see a list of the caregivers in the application. The app takes the availability of the caregivers into account through a colored phone icon. A green icon is shown when the caregiver is available. If the caregiver has indicated to be busy or unavailable, that specific caregiver will not be shown on the list. If no information is known in the system, a gray icon is shown.

\subsubsection{The Algorithm}

When the OCarePlatform decides an incident has occurred, the event handling process is activated. This process consists of several steps. Algorithm 1 explains the event handling process in pseudo-code.

First, the desktop operator checks whether an incident actually occurred and it was not a false alarm. If it concerned a false alarm, the information is logged (which in the future could be used to further enrich the reasoning process of the OCarePlatform) and the call between the call center and the older adult is ended. Next, using a checklist, it is determined whether there is a need for immediate assistance by an ambulance. This can be because of heavy bleeding, immobility of arms or legs, or the older adult is not answering, which may indicate unconsciousness. After this step, the location of the older adult is checked by the elderly app. If the fall victim is in his/her house, then key access may be necessary. This is important to take into account when selecting the most appropriate caregiver. The input from the caregiver app from the different (in)formal caregivers is analyzed to provide the desktop operator with a sorted list where the most appropriate caregiver to answer the call is ranked first. If nobody has access to the home and this is actually needed, the ambulance is notified. The desktop operator will notify the caregiver. The selected caregiver can indicate whether he/she is able to respond and how long it will take to go to the home of the older adult. The desktop operator will keep following up on the caregiver presence and the care giving during the intervention (minimally 1 update every 15 minutes). If the caregiver is not able to provide the necessary care, an additional person can be contacted. When the intervention has ended, the call is closed and the caregiver can fill in a small questionnaire about the provided care. This information will be logged.

\section{Results}

The process of the event handling system, using a social-aware selection of a caregiver to assist the older adult is based on the guidelines used in the current PERS. However, while using these guidelines as input for the FallRisk system, questions arose

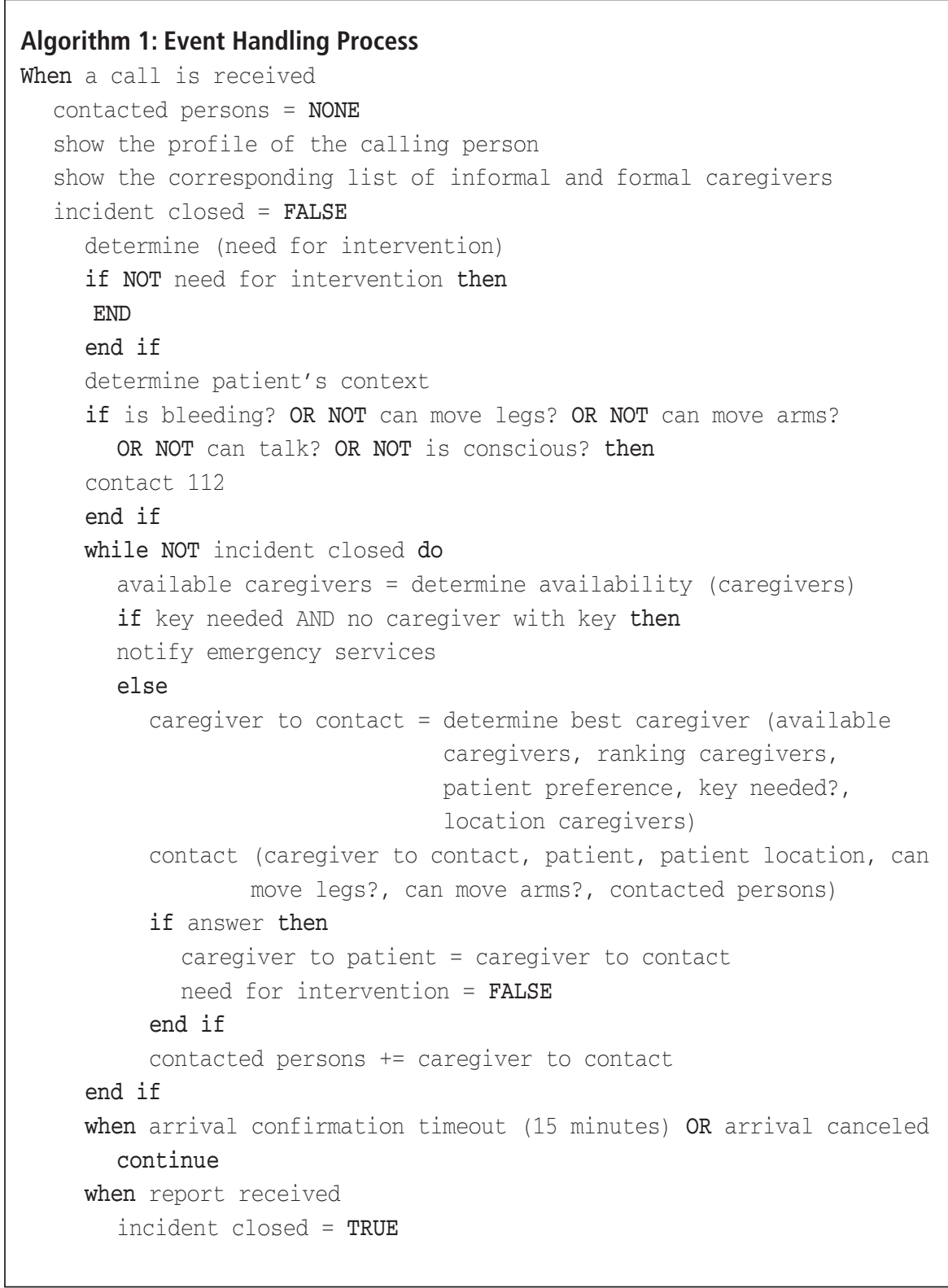


whether these guidelines were still up to date and whether changes, either big or small, were necessary. In this section, the evaluation of the event handling process is discussed.

\subsection{Evaluation Approach}

One way to evaluate the event handling process and to verify whether the steps defined within the algorithm are accepted by the domain experts and stakeholders, is to go directly to these end users to discuss and validate the process thoroughly. Domain experts can be identified as formal caregiver and the desktop operator, experienced in this domain, or for example, expertise centers, who create guidelines for specific process. Stakeholders can be defined as people who come in close contact with the system, such as the older adult and the informal caregivers. The reason to validate the event handling process in such a manner is because previous research revealed the AAL solutions do not take into account the requirements, needs and desires of the stakeholders and domain experts $[38,39]$. Without considering the input of these groups, the FallRisk system will never be accepted and used to facilitate independent living.

Previous research by Ongenae et al. [40] has revealed that traditional knowledge capturing techniques, such as stakeholder questionnaires and observations do not always reveal all necessary details to sufficiently understand the problem statement and underlying algorithmic dependencies to design an information system that end users really want to embrace. Therefore, a workshop, called a decision-tree workshop, can be organized in order to fill in the gaps, left open after the execution of the initial user research. To evaluate the event handling process, the user researchers and technical engineers, within the interdisciplinary FallRisk project, decided to organize such decision tree workshop. In this workshop, the knowledge of the domain experts and stakeholders can be captured and compared against the proposed process.

\subsection{Decision Tree Workshop}

The following subsections will discuss the preparation, objectives, participants and methods used within the workshop.

\subsubsection{Preparation}

In order to result in a successful experience for all participants, it is important that the workshop is well prepared and focused on one or more specific scenarios. In the case of fall handling and PERS, a number of characteristic and recognizable situations have been defined beforehand. In this case, the scenario was used where a fall incident occurs, as discussed in Section 3.2.

\subsubsection{Objectives}

An important goal of this workshop was the translation of the conceptual model into a formal one. This can be done through the definition of axioms which restrict the interpretation of the information in a given situation, e.g. when a fall incident needs to be treated as urgent and/or critical. Most of these restrictions were derived from the information obtained during the observations, which were organized in an earlier stage of the project.

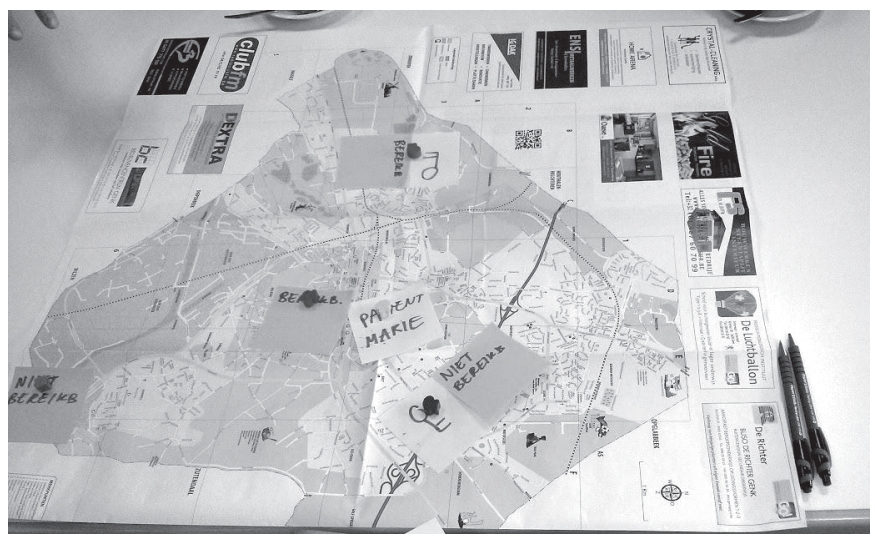

Figure 6 The map used during the decision tree workshop. This gives the participants a good indication of what needs to be known during the workshop and to have a physical overview of distance on the map.
The general goal of this type of workshop is to capture these decision processes, which can then be translated into additional ontology concepts and rules/axioms. In contrast to these general objectives, the main goal of this specific workshop at hand was not primarily to discover all logic and necessary information for the decision-making processes and workflows, but rather to check whether the existing guidelines really do reflect current day practices and requirements from all stakeholders. Of course, any missing information in the current guidelines can then be completed using the feedback from the participants during the workshop.

\subsubsection{Participants}

Five participants were recruited via a home care organization based on their different roles and tasks: one older adult using the PERS (F, 86), an informal caregiver (and also contact person) of the older adult (her daughter, 65) and three employees (of which two were nurses) working at the home care organization. Both nurses worked only part time as a home care nurse, while one (F, 35) worked part-time as an operator at the call center, answering calls from the PERS and the other (F, 54) was responsible for installing equipment, such as PERS systems, but also special beds or mattresses. The third employee (F, 29) was not a nurse, but was also responsible for installing equipment. The workshop lasted about 1.5 hours. Three researchers (2 user researchers and 1 engineer) were present to lead the workshop.

\subsubsection{Methods}

A detailed description of all methods used in decision tree workshop is given by Ongenae et al. [19]. However, for the convenience of the reader, a summary is presented.

First, the participants were asked to write down a number of situations they have come across and liked to see improved by the new PERS process. Then, during the main part of the workshop, they were asked to impersonate the actual intelligent all-knowing system. The aforementioned situations were selected to further discuss how this intelligent PERS ideally handles the situation. 
Each situation started with a very limited initial setup and was visualized on an easily understandable visualization, in this case, a city map, post-its and pawns, as shown in Figure 6 . To make a sensible decision, the participants playing the role of the system, ask for additional information about the situation by asking questions, e.g., "Do we know the personal details of the person who has fallen?", "Do we know who can be contacted?", "What are their roles?". Instead of immediately giving an answer, a discussion was encouraged about the importance of the requested information by asking three questions: (1) "Why do you feel the answer to this question is pertinent?", (2) "Does everyone agree?", (3) "Can you give examples of answers to this question?". Finally, the question was answered and visualized. Further questions unravel the situation. The questions and the order in which they were asked, give the researchers insights into the needed information and its importance for making a decision. The ontology engineer processed the outcome on paper in the form of a decision tree.

\subsection{Outcome and Results}

The outcomes described in this section, are based on watching and listening to the recorded footage of the workshop and the decision tree that was drawn during the workshop.

\subsubsection{Process}

At the start of the workshop, the participants were asked to write down a situation or experience they encountered with PERS. The nursing home employees seemed to understand the assignment immediately, while the informal caregiver was a bit reluctant to participate and had difficulties to write down a situation. Based on the response of the older adult, it was not clear if she understood the assignment, but she was able to tell about a situation in which she fell. One researcher worked together with the older adult to write down a particular situation, but she sometimes relied on her daughter to fill in some details, for instance, whether she already had the PERS at the moment of that particular fall.
When discussing which questions are asked first, all participants contributed, but some were more dominantly present than others. In the beginning, it had to be repeated and clarified that the questions were not asked to the older adult, but to the allknowing system. Especially the nurses who took more actively part in the discussion - had to be reminded repeatedly that the exercise was about the desired situation, which might be different from their current working process. When the participating older adult made remarks, it was often related to incidents that happened to herself.

\subsubsection{Results}

The workshop confirmed the wanted process largely corresponds to the process currently followed when a PERS call is received. On overview of the decision tree (in Dutch) drafted during the workshop is shown in Figure 7. The workshop, however, also resulted in qualitative insights; experiences of the persons participating in the workshop and possible opportunities, where sensor technology can help without disrupting the overall process.

- The availability of the caregivers is a crucial factor for which in the current PERS a manual process exits. However, this process only takes into account long periods of absence, such as vacations, and only if the caregiver reports this. In the current system, the order of the list is preset and thus the list is called in that order, which is very time-consuming and not efficient.

- Time to get to the older adult is also very important. Within the PERS, there is no knowledge about the caregivers' actual location, potentially leading to several wrong attempts as caregivers are no longer closely located to their home most of the time, which is the only information available at the moment.

- Location of the patient within the home or outside is also crucial to know. If the older adult is fallen in the home, it is important that the caregiver has access to the home.

- Also, it was evident that in the current working process, there was sometimes a 'struggle' between the wishes of the nurses and the PERS users. For instance, the older adult wanted to be contacted by a specific person (for instance due to fights with other contact persons), while the nurses thought it was more important that the person who is contacted, is closest in distance to the fall victim. However, because the nurses respect the wishes of the PERS users, the preferred contact person is contacted first. For instance, if they ask to call an ambulance, the nurse has to call an ambulance.

By using technology present in the smart devices, which are used in the FallRisk system, this information can easily be gathered and used within the event handling process and the current guidelines can be enhanced.

\section{Discussion}

In the following sections, the outcomes, involvement of the stakeholder and organization and preparation of the workshop are discussed.

\subsection{Workshop Outcomes}

The workshop was successful in gathering which information is needed during the process when the PERS is activated. Due to the nature of the workshop (an all-knowing being), we also learned when and why this information is required, but not how this information is obtained. The natural tendency of the involved users to base themselves on the existing process can be used to identify parts of which acceptance of a new solution may be difficult and may thus be used at a later point to ease migration to a new process.

Besides the need for information, we also found that the priorities of the various stakeholders are not always similar, but that certain decisions are made out of respect for the fall victim (e.g. contacting a specific person, or contacting an ambulance upon the request of the fall victim) and not necessarily because the situation requires this.

The workshop also provided insights about the diversity of priorities across stakeholders and how these are weighted to 
reach an agreement. The role of the PERS is to assist in the process, not to replace the final human decision maker.

In contrast to previous experience, the decision tree workshop was organized independently from all other knowledge gathering tasks within the FallRisk project. One important reason for this is that the ontology models, which have been engineered over time previously, are easily incorporated and minimally enhanced for the specific domain at hand. Restricting the interpretation of the concepts in the ontology, by means of axioms, was a part of the domain knowledge that had to be engineered for the specific setting of a PERS. A
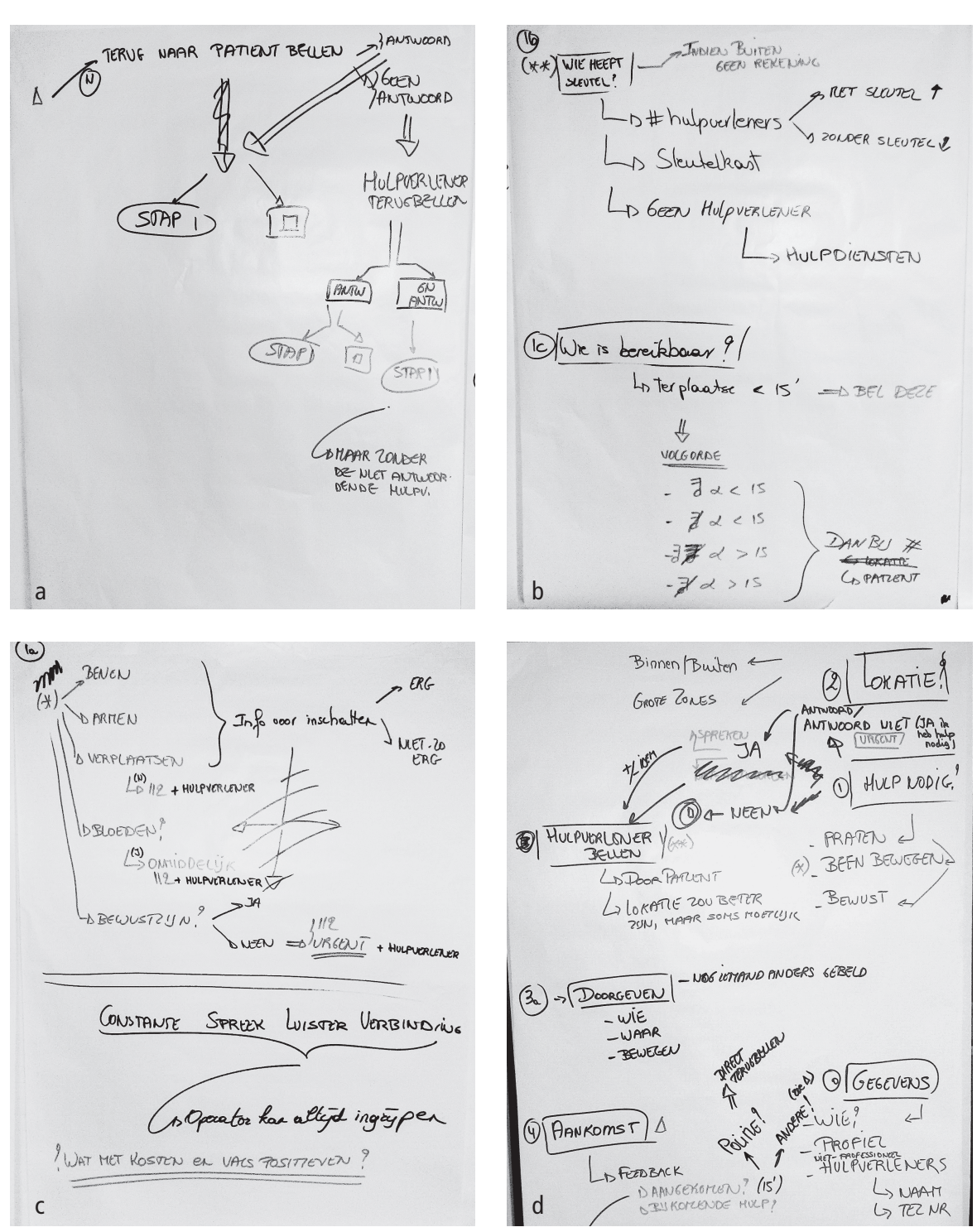

Figure 7 The results (in Dutch) of the decision tree workshop, which was created by the engineer during the discussions in the decision tree workshop.

decision tree workshop has proven to be the ideal tool to accomplish this task.

\subsection{Stakeholder Involvement}

A decision tree workshop is an opportunity for discussion with relevant stakeholders. However, the focus of the workshop was a specific process that mainly involved the operators (only 2-5 participants). As a consequence, the other participants had less to add to the workshop and the moderator had to pay attention to addressing the other participants to involve them in the workshop. Note that the format of the workshop facilitates this, an agreement is

\section{locat} pared to the fall victim. This ensured the same mental model of the proximity of the contact persons (visualized as pawns), and in addition, post-its were used to indicate whether these contact persons had key access to the fall victim's home.

\section{Conclusions}

In this paper, a social-aware event handling system is presented. When the OCarePlatform detects an incident, based multi-sensor fall detection, the desktop operator is notified and the event handling process is started. A detailed overview of the components of the FallRisk system and their interactions is given to have an overview of how the system works. The event handling process was based on the current guidelines and 
enhanced with extra information that nowadays can be gathered from smart devices, such as a smartphone, a tablet or a smart TV. To evaluate whether this process fulfilled the needs, a decision tree workshop was organized with stakeholders and domain experts. The results of this evaluation indicated that the proposed process was in line with the expectations, but that current guidelines get updated as technology keeps evolving. Future research will focus on the analysis of logged information to improve the back-end reasoning of the FallRisk system.

\section{Acknowledgment}

Companies and organizations involved in the project are COMmeto, Televic Healthcare, TP Vision, Verhaert and Wit-Gele Kruis Limburg, with project support of IWT.

\section{Conflict of Interest}

The authors declare that they have no conflict of interest.

\section{References}

1. Mortality and life expectancy statistics - Statistics Explained [cited 2015 Nov 1]. Available from: http://ec.europa.eu/eurostat/statistics-explained/ index.php/Mortality_and_life_expectancy_statistics.

2. Rechel B, Grundy E, Robine J-M, Cylus J, Mackenbach JP, Knai C, et al. Ageing in the European Union. Lancet. 2013; 381: 1312-1322. doi: 10.1016/ S0140-6736(12)62087-X.

3. Population structure and ageing - Statistics Explained [cited 2015 Sep 30]. Available from: http://ec.europa.eu/eurostat/statistics-explained/ index.php/Population_structure_and_ageing.

4. Healthy life years statistics - Statistics Explained [cited 2015 Sep 30]. Available from: http://ec.europa.eu/eurostat/statistics-explained/index.php/ Healthy_life_years_statistics.

5. Van der Vlies RD, Nauta J, Smit-Rietveld C. Checking dwelling performance for Aging-inPlace. Stud Health Technol Inform. 2015; 217: 1040-1046.

6. Davey JA, New Zealand Institute for Research on Ageing. Accommodation Options for Older People in Aotearoa/New Zealand. 2004.

7. Silva AL, Teixeira HJ, Teixeira MJC, Freitas S. The needs of informal caregivers of elderly people living at home: an integrative review. Scand J Caring Sci. 2013; 27: 792-803. doi: 10.1111/scs.12019.

8. Anderson WL, Wiener JM. The impact of assistive technologies on formal and informal home care. Gerontologist. 2015; 55: 422-433. doi: 10.1093/geront/gnt 165 .

9. Ambrose AF, Geet P, Hausdorff JM. Risk factors for falls among older adults: A review of the litera- ture. Maturitas. 2013; 75: 51-61. doi: 10.1016/ j.maturitas.2013.02.009.

10. Hawley-Hague $\mathrm{H}$, Helen $\mathrm{H}-\mathrm{H}$, Elisabeth B, Alex $\mathrm{H}$, Klaus P, Chris T. Older adults' perceptions of technologies aimed at falls prevention, detection or monitoring: A systematic review. Int J Med Inform. 2014; 83: 416-426. doi: 10.1016/j.ijmedinf.2014.03.002.

11. Belshaw M, Taati B, Snoek J, Mihailidis A. Towards a single sensor passive solution for automated fall detection. Conf Proc IEEE Eng Med Biol Soc. 2011; 2011: 1773-1776. doi: 10.1109/IEMBS.2011. 6090506.

12. Song Z, Zhenyu S, Lazarescu MT, Riccardo T, Luciano L, Spirito MA. High-Level Internet of Things Applications Development Using Wireless Sensor Networks. In: Mukhopadhyay SC, editor. Internet of Things, Springer; 2014. p. 75-109. doi: 10.1007/ 978-3-319-04223-7 4 .

13. Bardram JE. Pervasive healthcare as a scientific discipline. Methods Inf Med. 2008; 47(3): 178-185.

14. Habib MA, Mohktar MS, Kamaruzzaman SB, Lim KS, Pin TM, Ibrahim F. Smartphone-based solutions for fall detection and prevention: challenges and open issues. Sensors. 2014; 14: 7181-7208. doi: 10.3390/s140407181.

15. Arnrich B, Mayora O, Bardram J, Tröster G. Pervasive healthcare. Methods Inf Med. 2010; 49(1): 67-73.

16. Spitalewsky K, Rochon J, Ganzinger M, Knaup P. Potential and requirements of IT for ambient assisted living technologies. Methods Inf Med. 2013; 52(3): 231-238.

17. Rashidi P, Mihailidis A. A survey on ambient-assisted living tools for older adults. IEEE journal of biomedical and health informatics. 2013; 17(3): 579-590.

18. Ludwig W, Wolf KH, Duwenkamp C, Gusew N, Hellrung N, Marschollek M, Wagner M, Haux R. Health-enabling technologies for the elderly-an overview of services based on a literature review. Computer methods and programs in biomedicine. 2012; 106(2): 70-78.

19. Igual R, Medrano C, Plaza I. Challenges, issues and trends in fall detection systems. Biomedical engineering online. 2013; 12(1): 1.

20. Bagalà F, Becker C, Cappello A, Chiari L, Aminian K, Hausdorff JM, Zijlstra W, Klenk J. Evaluation of accelerometer-based fall detection algorithms on real-world falls. PloS one. 2012; 7(5): e37062.

21. Mubashir M, Shao L, Seed L. A survey on fall detection: Principles and approaches. Neurocomputing. 2013; 100: 144-152.

22. Chaudhuri S, Thompson H, Demiris G. Fall detection devices and their use with older adults: a systematic review. Journal of geriatric physical therapy (2001). 2014; 37(4): 178.1

23. Hawley-Hague H, Boulton E, Hall A, Pfeiffer K, Todd C. Older adults' perceptions of technologies aimed at falls prevention, detection or monitoring: a systematic review. International journal of medical informatics. 2014; 83(6): 416-426.

24. Rubenstein LZ. Falls in older people: epidemiology, risk factors and strategies for prevention. Age and ageing. 2006; 35(Suppl 2): ii37-41.

25. Lord SR, Sherrington C, Menz HB, Close JC. Falls in older people: risk factors and strategies for prevention. Cambridge; Cambridge University Press; 2007.

26. Learn not to Fall. How often Falls occur, 2012. Available from: http://www.learnnottofall.com/ content/fall-facts/how-often.jsp.
27. Abbate S, Avvenuti M, Bonatesta F, Cola G, Corsini P, Vecchio A. A smartphone-based fall detection system. Pervasive and Mobile Computing. 2012; 8(6): 883-899.

28. Sposaro F, Tyson G. iFall: an Android application for fall monitoring and response. In 2009 Annual International Conference of the IEEE Engineering in Medicine and Biology Society 2009 Sep 3 (pp. 6119-6122). IEEE.

29. Lee JV, Chuah YD, Chieng KT. Smart elderly home monitoring system with an android phone. Int J Smart Home. 2013; 7(3): 17-32.

30. Iqbal MU, Fet N, Wagner S, Handte M, Marrón PJ. Living++: A platform for assisted living applications. In Proceedings of the 2013 ACM conference on pervasive and ubiquitous computing adjunct publication 2013 Sep 8 (pp. 853-860). ACM.

31. Terroso M, Freitas R, Gabriel J, Marques AT, Simoes R. Active assistance for senior healthcare: A wearable system for fall detection. In 2013 8th Iberian Conference on Information Systems and Technologies (CISTI) 2013 Jun 19 (pp. 1-6). IEEE.

32. Marschollek M, Rehwald A, Wolf KH, Gietzelt M, Nemitz G, Meyer Zu Schwabedissen H, Haux R. Sensor-based fall risk assessment - an expert 'to go. Methods Inf Med. 2011; 50(5): 420-426.

33. Nelis J, Verschueren T, Verslyppe D, Develder C. DYAMAND: DYnamic, Adaptive MAnagement of Networks and Devices. 37th Annual IEEE Conference on Local Computer Networks, 2012. doi: 10.1109/lcn.2012.6423604.

34. De Backere F, Ongenae F, Van den Abeele F, Nelis J, Bonte P, Clement E, et al. Towards a social and context-aware multi-sensor fall detection and risk assessment platform. Comput Biol Med. 2015; 64: 307-320. doi: 10.1016/j.compbiomed.2014.12.002.

35. Ongenae F, Famaey J, Verstichel S, De Zutter S, Latré $S$, et al. Ambient-aware continuous care through semantic context dissemination. BMC Med Inform Decis Mak. 2014; 14: 97. doi: 10.1186/1472-6947-14-97.

36. Van den Bergh J, Elprama S, Luyten K, Aendekerk B, De Backere F, Jacobs A. Interdisciplinary Design of a Pervasive Fall Handling System. Proceedings of the 8th International Conference on Pervasive Computing Technologies for Healthcare, 2014. doi: 10.4108/icst.pervasivehealth.2014.255784.

37. Van den Bergh J, Elprama S, Decancq J, Jacobs A, Coninx K. Context Awareness in Communication around Fall Handling with PERS. INTERACT 2015 Adjunct Proceedings: 15th IFIP TC. 13 International Conference on Human-Computer Interaction 14-18 September 2015, Bamberg, Germany, vol. 22, University of Bamberg Press; 2015, p. 2015345.

38. Rashidi P, Mihailidis A. A survey on ambient-assisted living tools for older adults. IEEE journal of biomedical and health informatics. 2013; 17(3): 579-590.

39. Calvaresi D, Cesarini D, Sernani P, Marinoni M, Dragoni AF, Sturm A. Exploring the ambient assisted living domain: a systematic review. Journal of Ambient Intelligence and Humanized Computing. 2016: 1-9.

40. Ongenae F, Duysburgh P, Sulmon N, Verstraete M, Bleumers L, De Zutter S, et al. An Ontology Codesign Method for the Co-creation of a Continuous Care Ontology. Appl Ontol. 2014; 9: 27-64. 